This is the peer reviewed version of the following article: "Lana, $X$., Burgueño, A., Martínez, M.D. and Serra, C. (2016) Complexity and predictability of the monthly Western Mediterranean oscillation index. International Journal of Climatology, (36) 6: 2435-2450." which has been published in final form at [10.1002/joc.4503]. This article may be used for non-commercial purposes in accordance with Wiley Terms and Conditions for Self-Archiving." 


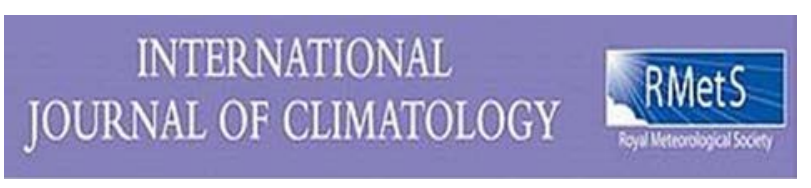

\section{COMPLEXITY AND PREDICTABILITY OF THE MONTHLY WESTERN MEDITERRANEAN OSCILLATION INDEX}

\begin{tabular}{|r|l|}
\hline Journal: & International Journal of Climatology \\
\hline Manuscript ID: & JOC-15-0152.R1 \\
\hline Wiley - Manuscript type: & Research Article \\
\hline Date Submitted by the Author: & 23-Jul-2015 \\
\hline Complete List of Authors: & $\begin{array}{l}\text { Lana, Xavier; Univ. Politècnica de Catalunya, Física i Enginyeria Nuclear } \\
\text { serra, carina; Universitat Politècnica de Catalunya, Fisica i Enginyeria } \\
\text { Nuclear } \\
\text { Martinez, M. Dolors; Univ. Politècnica Catalunya, Fisica Aplicada } \\
\text { Burgueño, August; Universitat de Barcelona, Astronomy and Meteorology }\end{array}$ \\
\hline Keywords: & $\begin{array}{l}\text { monthly Western Mediterranean Oscillation index, complexity and } \\
\text { predictive instability, fractional Gaussian noise simulation and } \\
\text { autoregressive process. }\end{array}$ \\
\hline &
\end{tabular}


${ }^{(1)}$ Lana, X., ${ }^{(2)}$ Burgueño, A., ${ }^{(3)}$ Martínez, M.D., ${ }^{(1)}$ Serra, C.,

(1) X. Lana, Serra C. Departament de Física i Enginyeria Nuclear. Universitat Politècnica de 9 Catalunya.

$10{ }^{(2)}$ A. Burgueño. Departament d'Astronomia i Meteorologia. Universitat de Barcelona.

${ }^{(3)}$ M.D. Martínez. Departament de Física Aplicada. Universitat Politècnica de Catalunya.

Corresponding autor: Xavier Lana 


\section{Abstract}

20 The complexity, predictability and predictive instability of the Western Mediterranean

21 Oscillation index at monthly scale, WeMOi, years 1856-2000, are analysed from the viewpoint

22 of monofractal and multifractal theories. The complex physical mechanism is quantified by: 1)

23 the Hurst exponent, $H$, of the rescaled range analysis; 2) correlation and embedding 24 dimensions, $\mu^{*}$ and $d_{E}$, together with Kolmogorov entropy, $\kappa$, derived from the 25 reconstruction theorem; and 3) the critical Hölder exponent, $\alpha_{o}$, the spectral width, $W$, and 26 the asymmetry of the multifractal spectrum, $f(\alpha)$. The predictive instability is described by 27 the Lyapunov exponents, $\lambda$, and the Kaplan-Yorke dimension, $D_{K Y}$, while the self-affine 28 character is characterized by the Hausdorff exponent, $H_{a}$. Relationships between the exponent $\beta$, which describes the dependence of the power spectrum $S(f)$ on frequency $f$, and the Hurst and Hausdorff exponents suggest fractional Gaussian noise, $\mathrm{fGn}$, as a right simulation of empiric WeMOi. Comparisons are made with monthly North-Atlantic Oscillation, NAO, and Atlantic Multidecadal Oscillation, AMO, indices. The analysis is complemented with an $\operatorname{ARIMA}(p, 1,0)$ autoregressive process, which yields a more accurate prediction of WeMOi than that derived from $f G n$ simulations.

Key words: Western Mediterranean Oscillation index (WeMOi), reconstruction theorem, complexity and predictive instability, multifractal spectrum, fractional Gaussian noise simulation, autoregressive process. 

1. Introduction

The Western Mediterranean Oscillation, WeMO, affects the climate variability in the eastern Iberian Peninsula, its effects being dominant in comparison with those of the North Atlantic Oscillation, NAO, and the Atlantic Multidecadal Oscillation, AMO, (Martín-Vide and LópezBustins, 2006; Mariotti and Dell'Aquila, 2012). It is assumed the hypothesis that WeMO, as the other two oceanic oscillations, is a complex signal without a regular behaviour, although not completely random. This hypothesis will be verified in agreement with fractal parameters obtained in here, which will quantify the complexity and predictive instability of WeMO. At the same time, it is assumed that WeMO derives from the nonlinear system of differential equations governing the atmospheric dynamics (Carlson, 1994; Holton, 2004; Martin, 2006; Mak, 2011) and the ocean-atmosphere interactions (Wells, 1986; Curry and Webster, 1999; Wallace et al., 1990; Barsugli and Battisti, 1998; Bhatt et al., 1998; Czaja and Frankignoul, 1999; Ciasto and Thompson, 2004; Mosedale et al., 2005, 2006; Xin et al., 2015; Goodman and Marshall, 1999; Latif, 1998).

Fractal analysis studies dynamics of nonlinear determinist signals, like these sea/oceanic oscillations. With the aim of deriving the main fractal properties of the monthly WeMO index, WeMOi, different measures of complexity are applied by assuming mono- and multifractality of the series. Monofractal series are homogeneous in the sense that they have self-similar properties throughout the entire time series, while multifractal series are locally self-similar, i.e. the property of self-similarity may be kept separately within different ranges. Both approaches are considered, with the aim of characterizing the complex and chaotic behaviour of the WeMOi. Results of the fractal analysis of the monthly WeMO, NAO (Martínez et al., 2010) and AMO indices are compared. Prediction of WeMOi is also attempted by means of two different strategies: first, simulations of fractional Gaussian noise, fGn, series, as suggested by monofractal parameters; and second, an $\operatorname{ARIMA}(p, 1,0)$ autoregressive process, improving previous $\mathrm{fG}$ results, which would permit replacing the complexity of the physical mechanism by a high order multilinear process. Additionally, cross-correlations and crosspower spectra results discard dependences of WeMOi on monthly NAO and AMO indices.

The contents of the paper include a description of the WeMOi, its cumulative distribution (Section 2), monofractal properties (Section 3) and multifractal characteristics derived from the multifractal detrended fluctuaction analysis, MF-DFA, which is also succinctly described (Section 4). A comparison of the mono- and multifractal properties for the WeMO, NAO and AMO indices, and the validity of $\mathrm{fGn}$ series to simulate the WeMOi are presented in Section 5. Section 6 introduces results derived from the ARIMA process and the Conclusions Section outlines the main WeMOi fractal features and reviews the autoregressive prediction results. 
International Journal of Climatology - For peer review only

Page 4 of 42

81

$$
\begin{aligned}
& 1 \\
& 2 \\
& 3 \\
& 4 \\
& 5 \\
& 6 \\
& 7 \\
& 8 \\
& 9 \\
& 10 \\
& 11 \\
& 12 \\
& 13 \\
& 14 \\
& 15 \\
& 16 \\
& 17 \\
& 18 \\
& 19 \\
& 20 \\
& 21 \\
& 22 \\
& 23 \\
& 24 \\
& 25 \\
& 26 \\
& 27 \\
& 28 \\
& 29 \\
& 30 \\
& 31 \\
& 32 \\
& 33 \\
& 34 \\
& 35 \\
& 36 \\
& 37 \\
& 38 \\
& 39 \\
& 40 \\
& 41 \\
& 42 \\
& 43 \\
& 44 \\
& 45 \\
& 46 \\
& 47 \\
& 48 \\
& 49 \\
& 50 \\
& 51 \\
& 52 \\
& 53 \\
& 54 \\
& 55 \\
& 56 \\
& 57 \\
& 58 \\
& 59 \\
& 60
\end{aligned}
$$

http://mc.manuscriptcentral.com/joc 


\section{The monthly Western Mediterranean Oscillation index, WeMOi}

83

84

The WeMOi was proposed by Martín-Vide and López-Bustins (2006) to detect atmospheric circulation patterns related to rainfall shortage or excess affecting the eastern Iberian Peninsula (Martín-Vide et al, 2008; López-Bustins et al., 2008; Gonzalez-Hidalgo et al., 2009), being also used in other regional climatic applications (Azorín-Molina and Lopez-Bustins, 2008; Sánchez-Lorenzo et al., 2009; Vicente-Serrano et al., 2009; Ouachani et al., 2013; El Kenawy et al., 2013; Beranová and Kyselý, 2015; Ríos-Cornejo et al., 2015). The WeMOi is defined as the difference between the normalized monthly barometric series at San Fernando (Spain) $\left(36^{\circ}\right.$ $17^{\prime} \mathrm{N}, 06^{\circ} 07^{\prime} \mathrm{W}$ ) and the normalized monthly barometric series at Padova (Italy) (45 $25^{\prime} \mathrm{N}$, $11^{\circ} 24^{\prime} \mathrm{E}$ ), with average and standard deviation being derived from the 1961-1990 period. In agreement with its definition, this atmospheric circulation index is expected to be strongly linked to Mediterranean climate patterns in contrast with others well known indices, as the NAO, with Atlantic climate influences. Positive phases of WeMOi are characterised by Azores anticyclone enclosing the south-west Iberian quadrant and low pressures at the Liguria Gulf. Negative phases are usually linked to Central Europe anticyclones (north of the Italian Peninsula) and low pressures at the south-west of the Iberian Peninsula. Neutral phases use to be coincident with north-eastern advections or low-pressure gradients over the western Mediterranean. Very illustrative examples of synoptic maps concerning these WeMOi phases can be found in Martín-Vide and López-Bustins (2006).

Figure 1a shows the time evolution of the WeMOi along the analyzed period (years 18562000). It is worth mentioning that extreme values of the WeMOi $(<5 \%$ and $\geq 95 \%)$ are approximately out of the \pm 2.1 range. The dashed line represents the time trend given by a third-degree polynomial fit, which depicts a negative slope since the beginning of the $20^{\text {th }}$ century and especially after 1950's. A deeper insight into this, by distinguishing between seasonal scales (Figure 1 b), strongly suggests that this decreasing tendency could be mainly linked to the behaviour of WeMOi at spring and summer seasons. The cumulative distribution of WeMOi (Figure 2) is Gaussian, the observations being kept within the $95 \%$ confidence bands derived from the Kolmogorov-Smirnov test (Benjamin and Cornell, 1970; Press et al., 1992). Figure 3 represents WeMOi for several return periods, given in months, compared with theoretic return values for a Gaussian distribution. Empiric values are also quite well described by a logarithmic law, by taking as argument the return period (months). Taking into account the symmetry of the Gaussian distribution, similar results would be obtained for negative WeMOi.

On the other hand, it is well known that the Mediterranean climate is submitted to the NAO (Trigo et al., 2002; Lionello et al., 2006; López-Moreno et al., 2011), weakly affecting the winter and spring precipitation in the eastern fringe of the Iberian Peninsula, with a negative

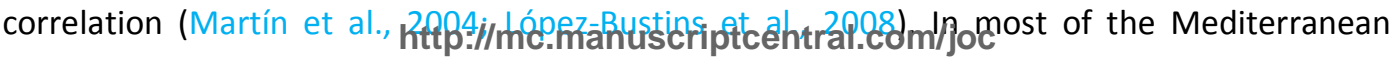


121 region, NAO plays a significant role on decadal variance in precipitation, especially for winter

122 (Mariotti and Dell'Aquila, 2012). Descriptions of the NAO dynamics and predictability can be

123 found in Jones et al. (1997), Hurrell et al. (2001), Fernández et al. (2003) and Collette and

124 Ausloos (2004), among others.

125

126 The AMO (also named "Atlantic Multidecadal Variability", AMV), is a signal defined from

127 North-Atlantic sea-surface temperatures (Enfield et al., 2001), which acts as a near-global

128 scale of multidecadal climate variability in the Northern Hemisphere (Knight et al., 2006;

129 Dijkstra et al., 2006; Dima and Lohmann, 2007). In the Mediterranean region at decadal time

130 scales and in summer months, the AMO has a large influence on anomalies on regional surface

131 air temperature and sea-surface temperature, accounting for over $30 \%$ of them. Significant

132 influence is also detected in the transition seasons, but not with precipitation (Mariotti and

133 Dell'Aquila, 2012).

134

135

http://mc.manuscriptcentral.com/joc 
The Hurst exponent, $H$, of the rescaled range analysis (Turcotte, 1997) is defined as the exponent of the power-law

$R(\tau) / S(\tau) \propto \tau^{H}$

being $R(\tau)$ the range of the different segments of length $\tau$ of a series and $S(\tau)$ the respective standard deviation. It should be remembered that $H$ close to 0.5 would imply a strong randomness of the series. Conversely, $H$ clearly lowering or exceeding 0.5 would suggest antipersistence or persistence respectively. Figure $4 a$ depicts the evolution of the quotient $R(\tau) / S(\tau)$ with $\tau$ in $\log$-log scales for the WeMOi. The computed $H$ value is close to 0.67 , thus pointing to a persistent signal, with a very good confidence level, as indicated by the square regression coefficient value $\left(\rho^{2}=0.998\right)$. Then, future WeMOi would partially depend on previous values, not necessarily in a linear form. Nevertheless time trends on previous values, among other factors, could be considered for improving predictions.

WeMOi autocorrelation, as a function of lag given in months, and its power spectrum are plotted in Figures $4 \mathrm{~b}$ and $4 \mathrm{c}$. Autocorrelation is characterised by a relatively narrow range $(-0.1,+0.2)$ and a clear periodicity close to 12 months. Additionally, given that notable correlations are not detected up to the maximum lag of 180 months, autoregressive processes would need a long monthly series to predict the next WeMOi. The spectral contents (Figure 4c) clearly show a periodicity of 1 year and two additional periodicities close to 19 and 51 years. The power spectrum amplitude $S(f)$ has a general decreasing trend with frequency, $f$, proportional to the power-law $f^{-\beta}$, with $\beta$ close to 0.17 , as estimated using the algorithm

The self-affine behaviour of a series can be verified according to two methodologies. First, in agreement with Turcotte (1997), a classic box-counting method can be applied to obtain the Hausdorff exponent, $H_{a}$. The fractal anisotropy of the series is assumed. This would imply that a certain property $g(x, y)$ in a two dimensional space is not statistically similar to $g(r x, r y)$, where $r$ is a scale factor, but similar to $g\left(r x, r^{H_{a}} y\right)$. And second, after applying the standard $\gamma(\tau)=\gamma_{0} \tau^{2 H a}$ in such a way that the evolution of $\gamma(\tau)$ with the different lengths $\tau$ on a log-log scale permits a straightforward evaluation of $H_{a}$. 
173

174

175

176

177

178

179

180

181

182

183

184

185

186

187

188

189

190

191

192

193

194

195

196

197

198

199

200

201

202

203

204

205

206

207

After determining empiric values of parameters $H, \beta$ and $H_{a}$, it can be assumed that WeMOi could be simulated by fGn (Turcotte, 1997). This hypothesis is based on the fact that $\mathrm{fGn}$ is compatible with Hausdorff exponents close to zero and $\beta$ parameters within the \pm 1 range. Moreover, $\mathrm{fGn}$ is compatible with a Hurst exponent close to 0.7 and the same range of $\beta$. $\mathrm{fGn}$ series may be then obtained after the following steps:

a) Generation of white Gaussian noise, wGn.

b) Discrete Fourier transform, DFT, of wGn.

c) Application of the filter $\left\{\frac{m}{N-1}\right\}^{\beta / 2}$ to the spectral contents of wGn, where $N$ is the number of samples of $w G n$ and $m$ the number of spectral frequencies.

d) The fGn series is finally generated by applying the inverse Fourier transform to the filtered wGn.

The similarity between the empiric WeMOi and the fGn series is assessed in Section 5.2, jointly with the same type of simulation for empiric monthly NAO and AMO indices.

Whereas the previous monofractal analyses have permitted to obtain a simulated model for the WeMOi, the reconstruction theorem (Diks, 1999) permits quantifying its complex predictability through a set of parameters as: the minimum number of nonlinear equations describing a physical mechanism, also named correlation dimension, $\mu(m)$, with $m$ the reconstruction space dimension; the embedding dimension, $d_{E}$, required to obtain an asymptotic value of the correlation dimension, $\mu^{*}$; and the Kolmogorov entropy, $\kappa$, which is a measure of the loss of memory of the physical process with time. The reconstruction theorem process is based on the generation of a set of $\mathrm{m}$-dimensional space vectors:

$z(i)=\{x(i), x(i+1), \ldots, x(i+m-1)\}, \quad i=1, \ldots, N$

and the definition of the correlation integral in terms of the Grassberger-Procaccia formulation (Grassberger and Procaccia, 1983a,b)

$C(m, r)=\lim _{N \rightarrow \infty} \frac{1}{N^{2}} \sum_{i, j=1}^{N} H\{r-\|z(i)-z(j)\|\}$

being $r$ an Euclidean distance in the $m$-dimensional space and $H\{$.$\} the Heaviside function.$ Additionally, it is assumed that

$C(m, r)=A_{m} e^{-m \kappa} r^{\mu(m)}$

with $\mu(m)$ the correlation dimension. After plotting the correlation integral (Equation $5, a$ ) in terms of $r$ on log-log scales

$\log \{C(m, r)\}=\log \left(A_{m}\right)-m \kappa+\mu(m) \log (r)$

the corresponding slope is $\mu(m)$ for every dimension $m$. Two factors have to be carefully reviewed, as they could lead to wrong $\mu(m)$ estimations: the lacunarity (Turcotte, 1997) for small values of $r$, and the saturation of $C(m, r)$ for high values of $r$, whatever the dimension $m$. 
208

209

210

211

212

213

214

215

216

217

218

219

220

221

222

223

224

225

226

227

228

229

230

231

232

233

234

235

236

237

238

239

240

241

242

An example for several reconstruction dimensions $m$ is depicted in Figure $5 a$, where the almost flat evolution of $C(m, r)$ for low values of $r$ and the saturation for high values of $r$ are quite evident. Nevertheless, although increasing values of $\mu(\mathrm{m})$ are derived for reconstruction dimensions up to 20 , they are found to tend towards an asymptotic value, $\mu^{*}$, close to 10.0 (inner plot in Figure 5a). This $\mu^{*}$ value provides a first evaluation of the complexity of the signal, as it indicates the minimum number of nonlinear equations required to describe the physical mechanisms governing the WeMOi. The random component of the WeMOi is also manifested by the high reconstruction dimension, $m=19$ or 20 , needed to obtain asymptotic values of the correlation dimension, $\mu^{*}$. This value is also known as embedding dimension, $\mathrm{d}_{\mathrm{E}}$, of the analysed series.

Another relevant feature of the reconstruction theorem is the quantification of the loss of memory of the physical system with time, through the Komogorov entropy, $\kappa$. Prediction of accurate future WeMOi will be difficult if $\kappa$ reaches a high value. Naming $\alpha(m)$ the term $\log \{C(m, r)\}-\mu(m) \log (r)$ in Equation $(5, \mathrm{~b})$, it results in

$\alpha(m)=\log \left(A_{m}\right)-m \kappa$

Equation $(5, c)$ permits to make an easy estimation of $\kappa$ by a least square regression of empiric $\alpha(m)$ in terms of $m$, provided that $\log \left(A_{m}\right)$ is constant. This constraint is only achieved for $m$ tending to $\infty$, for which it is expected that $A_{m+1} / A_{m}$ tends to 1.0 . This behaviour is shown in Figure 5b, where the Kolmogorov entropy is estimated to be close to 0.64 , by taking into account the linear evolution of $\alpha(m)$ for $m$ ranging from 16 to 20. If lower dimensions $m$ are considered, linearity disappears.

The last relevant application of the reconstruction theorem is the quantification of the predictive instability of the WeMOi through the Lyapunov exponents and the Kaplan-Yorke dimension. Considering the $m$-dimensional vectors of the reconstructed space generated according Equation (3) and in agreement with Wiggins (2003), the Lyapunov exponents, $\lambda_{j}(j=1,2, \ldots, m)$, can be computed according to the algorithms proposed by Eckmann et al. (1986) and Stoop and Meier (1988). Assuming that the addition of all the $m$ Lyapunov exponents is positive, the trajectory in the $m$-dimensional space will consist on aperiodic orbits around a strange attractor with a Kaplan-Yorke dimension, $D_{K Y}$ (Kaplan and Yorke, 1979), which is computed as

$D_{K Y}=\ell_{0}+\frac{1}{\left|\lambda_{\ell_{0}+1}\right|} \sum_{j=1}^{\ell_{0}} \lambda_{j}$

with $\ell_{0}$ the maximum number of Lyapunov exponents, in decreasing order, accomplishing $\lambda_{1}+\lambda_{2}+\ldots .+\lambda_{\ell_{0}} \geq 0$. Figures $6 a$ and $6 \mathrm{~b}$ show the evolution of the first two Lyapunov 

exponents towards asymptotic values, after a high enough number of iterations of the above

244 mentioned algorithms and for a sufficiently high reconstruction dimension $\mathrm{m}$. It is worth 245 mentioning that a minimum of 500 iterations is necessary and that the reconstruction theorem 246 has to be applied at least up to $m=14$ or 15 . A value of 0.16 is derived for $\lambda_{1}$. It has to be 247 underlined that predictive instability is assured if the first Lyapunov exponent is positive. 248 Additionally, the above mentioned strange attractor is characterized by a fractal structure with 249 $D_{K Y}$ equal to 12.43 , according to Equation (6).

250

251 


\section{Multifractal Analysis}

253

254

255

256

257

258

259

260

261

262

263

264

265

266

267

268

269

270

271

272

273

274

275

276

277

278

279

280

281

282

283

284

285

\subsection{Multifractal detrended fluctuation analysis, MF-DFA}

The MF-DFA has been introduced as a reliable characterization of multifractal non-stationary and stationary time series (Kantelhardt et al., 2002), and it is based on the identification of the scaling of the q-order moments depending on the signal length. The MF-DFA surpasses in quality and simplicity previous algorithms such as the multifractal box-counting (MF-BOX) (Feder, 1988) or the wavelet transform modulus maxima (WTMM) algorithms (Muzy et al., 1994). The MF-DFA has been applied in many scientific fields such as biology (Dutta, 2010), human health (Shimizu, 2002), seismology (Ghosh et al., 2012) or climatology (Feng et al., 2009; Burgueño et al., 2014; Mali, 2014). The steps for applying the MF-DFA can be summarised as follows:

1. Determination of the "profile" of the time series.

$Y(i) \equiv \sum_{k=1}^{i}\left[x_{k}-\langle x\rangle\right], \quad i=1, \ldots, N$

where $\langle x\rangle$ is the average value of the series.

2. Division of the profile $Y(i)$ into $N_{s} \equiv \operatorname{int}(N / s)$ non-overlapping segments of equal length $s$. Since the length $N$ of the series is often not a multiple of the considered timescale $s$, a short part at the end of the profile may remain. In order to not disregard this part of the series, the same procedure is repeated starting from the opposite end, thereby obtaining $2 N_{s}$ segments.

3. Computation of the local variance for each of the $2 N_{s}$ segments by a least-square polynomial fit of the series

$F^{2}(s, v) \equiv \frac{1}{s} \sum_{i=1}^{s}\left\{Y[(v-1) \cdot s+i]-y_{v}(i)\right\}^{2}$

for each segment $v, v=1, \ldots, N_{s}$, and

$F^{2}(s, v) \equiv \frac{1}{s} \sum_{i=1}^{s}\left\{Y\left[N-\left(v-N_{s}\right) \cdot s+i\right]-y_{v}(i)\right\}^{2}$

for $v=N_{s}+1, \ldots, 2 N_{s} \cdot y_{v}(i)$ is the fitting polynomial in segment $v$. The order of the polynomial has been found not to alter the results, this order varying from 2 to 5 (KoscielnyBunde et al., 2006). A fourth-order polynomial has been used in this study. This step assures the removal of non-stationarity from the $x_{k}$ series.

4. Average over all segments to obtain the qth-order fluctuation function, defined as:

$F_{q}(s) \equiv\left\{\frac{1}{2 \cdot N_{s}} \sum_{v=1}^{2 \cdot N_{s}}\left[F^{2}(s, v)\right]^{q / 2}\right\}^{1 / q}, q \neq 0, q \in \Re$

The interval $-15.0 \leq q \leq+15.0$, bar zero, with step 0.5 , has been considered here. 
of application,

$289 F_{0}(s) \equiv \exp \left\{\frac{1}{4 \cdot N_{s}} \sum_{v=1}^{2 \cdot N_{s}} \ln \left[F^{2}(s, v)\right]\right\}$

290

291

292

293

294

295

296

297

298

299

300

301

302

303

304

305

306

307

308

309

310

311

312

313

314

315

316

317

318

319

320

Steps 2 to 5 have to be repeated for several time scales s. Kantelhardt et al. (2002) have suggested values of $s$ in the interval $m+2 \leq s<N / 4$, where $m$ is the order selected for polynomial $y_{v}(i)$

6. Determination of the scaling behavior of the fluctuation functions by analyzing log$\log$ plots of $F_{q}(s)$ versus $s$ for each value of $q$. If the series $x_{i}$ are long-range power-law correlated, $F_{q}(s)$ increases, for large values of $s$, as a power-law

$$
F_{q}(s) \approx s^{h(q)}
$$

In general, the exponent $h(q)$ may depend on $q$. When the analyzed time series is nonstationary or noisy, such as fractional Brownian walks (Turcotte, 1997), the exponent $h(q=2)$ is larger than unity and satisfies $h(2)=H+1$, where $H$ is the well-known Hurst exponent (Movahed and Hermanis, 2008; Ge and Lung, 2012). For stationary time series, the exponent $h(2)$ is identical to the Hurst exponent $H$ (Feng and Xu, 2012). Then, the exponent $h(q)$ is known as the generalized Hurst exponent.

For monofractal time series, $h$ is independent of $q$, since the scaling behavior of the variances $F^{2}(s, v)$ is identical for all segments $v$. For multifractal time series, if we consider positive values of $q$, the segments $v$ with large variance $F^{2}(s, v)$ (i.e. large deviations from the corresponding fit) will dominate the average $F_{q}(s)$. Thus, for positive values of $q, h(q)$ describes the scaling behavior of the segments with large fluctuations. For negative values of $q$, the segments $v$ with small variance $F^{2}(s, v)$ will dominate the average $F_{q}(s), h(q)$ thus describing the scaling behavior of the segments with small fluctuations (Movahed and Hermanis, 2008).

\subsection{Singularity spectrum of the WeMOi}

According to Kantelhardt et al. (2002), the singularity spectrum $f(\alpha)$ can be related to the generalized Hurst exponent, $h(q)$, of the $q$ order fluctuation function, $F_{q}(s)$, via a Legendre transform

$\alpha=h(q)+q \frac{d[h(q)]}{d q} \stackrel{\text { Legendre }}{\leftrightarrow} f(\alpha)=q[\alpha-h(q)]+1$ 
where $\alpha$ is the singularity strength or Hölder exponent, while $f(\alpha)$ denotes the dimension

322 of the subset of the series. The multifractal scaling exponent is

$323 \tau(q)=q h(q)-1$

324

$\alpha$ being expressed as

$325 \quad \alpha=\frac{d \tau(q)}{d q}$

326

327

328

329

330

331

The characteristics of the singularity spectrum $f(\alpha)$ provide a new way of comparing signals, because $f(\alpha)$ describes the dimensions of subsets of the series characterized by the same singularity strength $\alpha$. Designing $\alpha_{o}$ as the singularity strength with maximum spectrum or critical Holder exponent, a small value of $\alpha_{o}$ means that the underlying process "loses finestructure"; that is, it becomes more regular in appearance, while a large value of $\alpha_{o}$ ensures larger complexity. In this sense, the Hurst exponent can be roughly related to the position of $\alpha_{o}$ (see for instance Burgueño et al., 2014). The shape of $f(\alpha)$ may be fitted to a quadratic function around the position $\alpha_{o}$,

$$
f(\alpha)=A\left(\alpha-\alpha_{0}\right)^{2}+B\left(\alpha-\alpha_{0}\right)+C
$$

where $C$ is an additive constant equal to 1 . Coefficient $B$ indicates the asymmetry of the spectrum, being zero for a symmetric spectrum. A right-skewed spectrum, $B>0$, indicates relatively strongly weighted high fractal exponents (with "fine-structure"), while left-skewed shapes, $B<0$, point to lower ones (more regular or smooth looking). The width of the spectrum, which is used as a measure of width of singularity strength range in the series, can be obtained by extrapolating the fitted curve to zero. Width $W$ is defined as

$$
W=\alpha_{1}-\alpha_{2}
$$

with $f\left(\alpha_{1}\right)=f\left(\alpha_{2}\right)=0$, being $\alpha_{1}$ larger than $\alpha_{2}$, and the wider the range of the Hölder exponent, the stronger the multifractality. In other words, the wider the range of $\alpha$, the "richer" is the process in structure. A signal with a high value of $\alpha_{o}$, a wide range of fractal exponents and a right-skewed shape is more complex than a signal with the opposite characteristics (Shimizu et al., 2002). Consequently, the fine-structure of physical mechanisms governing a signal could be analyzed if its complexity is high. On the contrary, if the signal has low complexity, only the smooth-structure of these mechanisms could be detected. For monofractal series, the width of the spectrum is zero and $h(q)$ is independent of $q$. Hence, from Equation (9), it is clear that there would be a unique value of $\alpha$ and $f(\alpha)$, the value of $\alpha$ being the Hurst exponent $H$ and the value of $f(\alpha)$ being equal to 1 . 
352 The results of applying MF-DFA to WeMOi are summarized in Figure 7. The power-law 353 behavior for $F_{q}(s)$ with $q=-15,0,+15$ is shown in Figure 7 a. In spite of some clear

354 fluctuations and disturbances, $F_{q}(s)$ depicts a power-law increasing with $s$. According to 355 Figures $7 \mathrm{~b}, 7 \mathrm{c}$ and $7 \mathrm{~d}$, the dependence of $h, \alpha$ and $\tau$ on $q$ is very well described by polynomial 356 relationships. The multifractal spectrum shown in Figure $7 \mathrm{e}$ is characterized by a moderate 357 asymmetry $(B=1.01)$, a critical Hölder exponent $\alpha_{o}$ equal to 0.57 , and a spectral width $W$ 358 equal to 0.23 , being $\alpha_{1}=0.70$ and $\alpha_{2}=0.47$. It is worth mentioning the very good fit of the 359 empiric spectrum to the $2^{\text {nd }}$ degree polynomial of Equation 15.

360 


\section{Comparisons of WeMOi to monthly NAO and AMO indices}

362

363

364

365

366

367

368

369

370

371

372

373

374

375

376

377

378

379

380

381

382

383

384

385

386

387

388

389

390

391

392

393

394

395

396

397

398

\subsection{Mono-and multifractal properties}

Table 1 summarises mono- and multifractal results obtained for monthly WeMO, NAO (Martínez et al., 2010) and AMO indices. Some common mono- and multifractal patterns can be detected for the three indices, while others are quite different. The interpretation of Hurst exponents, $H$, could be sometimes debatable, given that mono- and multifractal techniques could lead to slightly different estimations of $H$. Nevertheless, the results derived for monthly NAO and AMO indices from both methods are essentially coherent, while those for WeMOi are a little more dissimilar. WeMOi shows moderate persistence from the viewpoint of rescaled analysis and randomness ( $H \approx 0.5$ ) from multifractality. From both viewpoints, monthly NAO index is clearly characterised by randomness and monthly AMO index by a strong persistence. Given that the Hurst exponents derived from multifractal analysis does not exceed 1.0 for any of the three monthly series, their stationary character should be accepted and simulations based on fractional Brownian walks should be then discarded. Alternatively, empiric signals could be well reproduced by $f G n$ or almost pure wGn. In spite of the different persistent or random character of the three series, their self-affine nature, characterised by their Hausdorff exponents, $H_{a}$, would not be a differentiating factor among them, as $H_{a}$ always varies within a narrow range from 0.05 to 0.10 . The exponent $\beta$ of the power-law governing the power spectrum slope is in agreement with the persistence and randomness of the three monthly indices. Whereas monthly AMO index is again characterised by long and strong persistence ( $\beta$ slightly exceeding 1.0), the moderate persistence of WeMOi is confirmed by a low value of $\beta$. The strong randomness of the monthly NAO index is suggested by a value of $\beta$ very close to zero, which would be coherent with a model close to a wGn.

In terms of the reconstruction theorem, according to the required minimum number of nonlinear equations, $\mu^{*}$, the complexity of the physical mechanism governing the time evolution of the three indices is very clear for monthly WeMO and NAO indices $\left(\mu^{*} \approx 10\right)$, and more moderate for monthly AMO index $\left(\mu^{*} \approx 7\right)$. Another notable question is the loss of memory of the physical mechanism with time. The highest Kolmogorov entropy $(\kappa=1.37)$ corresponds to monthly NAO index, possibly due to its dominant random character, while monthly WeMO and AMO indices, especially the latter, are characterised by not so high loss of memory. In spite of the different patterns derived from the reconstruction theorem for the three indices, their predictive instability is characterised by very similar first Lyapunov exponents, $\lambda_{1}$, and Kaplan-Yorke dimensions, $D_{K Y}$. Consequently, the magnitude of the predictive errors will depend in a similar way on the starting value uncertainty for the three indices. Additionally, orbits in the $m$-dimensional reconstruction space around the strange attractor should be very similar. 
399

400

401

402

403

404

405

406

407

408

409

410

411

412

413

414

415

416

417

418

419

420

421

422

423

424

425

426

427

428

429

430

431

432

433

434

435

From the point of view of the multifractal results, it is worth mentioning the fine-structure of the monthly AMO index, characterized by the highest critical Hölder exponent, $\alpha_{0}$, the highest positive asymmetry, $B$, and the wider multifractal spectrum content, $W$. Consequently, more detailed descriptions of the physical mechanisms governing the monthly AMO index are expected in comparison with WeMOi and, especially, monthly NAO index. WeMOi is characterised by smaller values of $\alpha_{0}, B$ and $W$ parameters than monthly AMO index. Monthly NAO index should be associated with a smooth-structure, characterised by null asymmetry and an almost monofractal character, manifested by an almost null multifractal spectrum width.

\section{$5.2 \mathrm{fGn}$ simulations}

Given that the three sets of Hurst, Hausdorff and $\beta$ parameters would be compatible with a $\mathrm{fGn}$, with the special case of the monthly NAO index close to a pure Gaussian noise $(\beta \approx 0)$, comparisons are made between empiric signals and synthetic $\mathrm{fGn}$ models generated with their corresponding $\beta$ values. Figure 8 shows the three signals compared with their synthetic simulations, good coincidence being observed. Nevertheless, it is advisable to quantify in some way the similarity between simulated and empiric monthly series. This can be made in two steps.

a) In agreement with Stephenson et al. (2000), the mean absolute deviation, MAD,

$$
M A D=\frac{1.483}{N} \sum_{j=1}^{N}|m(j)-s(j)|
$$

is applied, being $m(j)$ any of the three empiric monthly series, $s(j)$ the corresponding simulated series and $N$ the signal length .

b) If MAD is less than or equal to one standard deviation of $m(j)$, the simulated $f G n$ could be assumed compatible with the empiric series. Obviously, this possibility would not imply that the simulated series is necessarily a good prediction of the empiric one, month by month. It would be only established that the self-affine character, persistence or randomness and the power spectral contents are similar.

Table 2 summarises some statistical patterns of the differences between empiric and $\mathrm{fGn}$ series. It is worth mentioning that the three series of differences are distributed according a Gaussian model, this fact confirmed by the Kolmogorov-Smirnov test. It has to be also underlined that the standard deviations of the empiric monthly NAO and AMO indices are close to their MAD. Conversely, the MAD for WeMOi is notably higher than the corresponding standard deviation of the empiric values.

\subsection{Cross-correlations}

The cross-correlation and cross-power spectrum are analysed by pairs WeMO-NAO and WeMO-AMO indices, looking for possible linkages between them. Figure 9 depicts the main http://mc.manuscriptcentral.com/joc 
436 characteristics of both pairs. With respect to the WeMO-AMO pair, the cross-correlation 437 coefficients are very small, and the expected periodicity of one year is clearly observed in the 438 cross-power spectrum. It is also remarkable that half-year periodicity is not relevant and that 439 some relationship between both monthly signals could be assumed for a long period close to 44051 years, in agreement with the evolution of the cross-correlation along the months. It is also 441 worth mentioning that the power spectrum slope is well reproduced by a power-law with an 442 exponent $\beta$ equal to 0.81 . The pair WeMO-NAO is characterised by a range of cross443 correlation coefficients slightly wider than that corresponding to the WeMO-AMO pair. 444 Remarkable periodicities, in some way expected, of one year and half-year, and an almost null 445 slope for the power spectrum amplitude, with $\beta$ equal to 0.14 , are observed. As a summary, 446 the three monthly indices show some common features from several mono-multifractal points 447 of view, but a clear functional relationship is not found, except for half-year (pair WeMO-NAO) 448 and one-year (pairs WeMO-NAO and WeMO-AMO) periodicities. 


\section{The autoregressive process}

451

452

453

454

455

456

457

458

459

460

461

462

463

464

465

466

467

468

469

470

471

472

\subsection{Mathematical formulation}

The autoregressive integrated moving average $\operatorname{ARIMA}(p, d, 0)$ model (Box and Jenkins, 1976) assumes that

$\Delta^{d} x(i)=\theta+\mu x(i-1)+\sum_{k=1}^{p} \delta_{k} \Delta^{d} x(i-k)+a_{i} \quad(i=p+2, \ldots, N)$

Where $\{x\}$ is a set of $N$ empirical data and $\Delta x$ is the set of first differences $\Delta x(i)=[x(i+1)-x(i)]$, with $\Delta^{d} x(i-k)=[x(i-k+1)-x(i-k)]^{d} .\left\{\theta, \mu, \delta_{1}, \ldots, \delta_{p}\right\}$ are the parameters of the autoregressive process of order $p,\{a\}$ is a noise series and $d$ is a real number. Alternatively, the $\operatorname{ARIMA}(\mathrm{p}, \mathrm{d}, 0)$ model, with $d=1.0$, can be written as

$$
x(i)=\theta+\sum_{k=1}^{p} \delta_{k} x(i-k)+a_{i} \quad, i=p+1, \ldots, N
$$

where the time series $\{x\}$ is directly used instead of first differences. With the aim of avoiding singularities in the linear equation system used to estimate $\left\{\theta, \mu, \delta_{1}, \ldots, \delta_{p}\right\}$, parameter $\mu$ is implicitly included in parameter $\delta_{1}$. Equation (18b) is usually designed as autoregression, $A R(p)$. The resulting system of linear equations, disregarding the stochastic component $\{a\}$, can be represented in matrix form by

$Z=A W$

with $Z$ the $\{x(p+1), x(p+2), \ldots, x(n)\}$ vector, $n$ the number of empiric elements belonging to series $\{x\}$, and the $(n-p-1, p+1)$ matrix $A$ multiplying a $p+1$ dimension vector $W$ containing the parameters $\left\{\theta, \mu, \delta_{1}, \ldots, \delta_{p}\right\}$ to be solved from Equation (19a). The components of vector $W$ can be estimated by multiplying Equation (19a) by the transposed $A$ matrix, $A^{T}$

$$
A^{T} Z=A^{T} A W
$$

Remembering that the symmetric matrix $A^{\top} A$ can be decomposed in two triangular matrices, it is straightforward to obtain the values of parameters $\left\{\theta, \delta_{1}, \ldots, \delta_{p}\right\}$ taking advantage of the Crout's algorithm (Press et al., 1992).

A convincing solution of Equation (18b) demands some criterion to decide the optimum autoregression order, OAO. The decision can be taken by looking for the order leading to the minimum of MAD (Equation 17).

\subsection{Results of the autoregressive process.}

The evolution of the MAD with the autoregressive order $p$ is depicted in Figure 10a. In spite of the wide range of $p$ analyzed and the decreasing tendency on MAD with increasing $p$, the reduction of the MAD is persistent but small, being finally chosen 145 as OAO, which corresponds to the lowest achieved value of MAD after exploring it from $p$ equal to 2 to 200 .

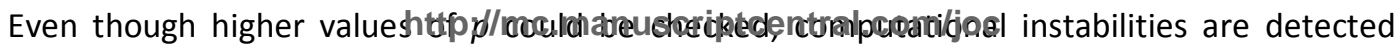


when the Crout's algorithm is applied to solve Equation 18b for autoregressive orders

486 exceeding $p=200$.

487

488

489

Figure $10 \mathrm{~b}$ depicts the time evolution of the autoregressive process residuals, which are quantified as the difference between a predicted WeMOi and the corresponding empiric value. 490 Positive (negative) residuals represent overestimation (underestimation) of WeMOi. Bearing in 491 mind the standard deviation of the real WeMOi $\left(\sigma_{\text {Wemo }}=1.17\right)$ and of the residuals $\left(\sigma_{\text {res }}=\right.$ 492 1.04), 63.5\% of monthly WeMOi are predicted with over- or underestimation smaller than the 493 original data standard deviation. This percentage corresponds to residuals included within the $494\left(-\sigma_{\text {res }}+\sigma_{\text {res }}\right)$ interval. Extreme residuals (below $-2.0 \sigma_{\text {res }}$ or above $\left.2.0 \sigma_{\text {res }}\right)$ correspond to a 495 percentage of $4.2 \%$. These last cases should be assumed as WeMOi predictions with excessive 496 errors.

497

498 Figure 10c shows the histogram of WeMOi residuals. First of all, it is worth mentioning the 499 relatively wide range of residuals, within $\pm 3 \sigma_{\text {res}}$, although mostly distributed within the $\pm 1.0 \sigma_{\text {res }}$ 500 interval. Second, residuals follow a Gaussian distribution (average almost equal to 0.0 and 501 standard deviation equal to 1.04), given that the Kolmogorov-Smirnov test is accomplished 502 with $95 \%$ of confidence. Consequently, under- and overestimations are expected to be very 503 balanced on the WeMOi predicted by the autoregressive process. Additionally, biases on the 504 WeMOi predictions are not to be expected, given the almost null residual average. 505 506 
The WeMOi is characterised by signs of moderate persistence, in contrast with a clear random behaviour of monthly NAO index and a strong persistence of monthly AMO index. The persistent character of WeMOi would suggest that successful prediction of forthcoming monthly values would be partially based on considering previous time trends. Nevertheless, results obtained from the reconstruction theorem put the stress on the complex physical system governing WeMOi. First of all, in agreement with the obtained asymptotic correlation dimension, $\mu^{*}$, a high number of nonlinear equations would be required to describe the physical mechanism. Second, the loss of memory, quantified by the Kolmogorov entropy, is notable. Consequently, the success of a predictive process will not be assured by taking into account a short set of previous monthly samples. Third, the predictive instability is made evident by the existence of positive Lyapunov exponents. In this way, long-term predictions would be strongly affected by small uncertainties at the beginning of the predictive process. Additionally, bearing in mind that the addition of the whole set of Lyapunov exponents is negative, WeMOi time evolution on a space of embedding dimension $d_{E}$ would be described by aperiodic orbits around a strange attractor of dimension $D_{K Y}$. For monthly NAO and AMO indices, the predictive instability and dimensions of the strange attractors are very similar to those of the WeMOi. Nevertheless, finding the appropriate predictive model is a bit more difficult for monthly NAO index. Comparatively, it requires the largest number of nonlinear equations and its loss of memory is the highest.

From the point of view of the physical mechanisms governing the three indices, it is outstanding the role of the large thermal inertia of oceanic water masses, which would explain high persistence and moderate loss of memory of monthly AMO index, in agreement with Kolmogorov entropy, $\kappa$, results. Conversely, the atmospheric pressure at sea level should be notably conditioned by the lower thermal inertia of the atmospheric air masses. This contrast of thermal inertia between oceanic and atmospheric masses could be assessed by the fact that the ocean thermal capacity is approximately equivalent to $\mathbf{3 8}$ atmospheric masses, when considering only the upper $100 \mathrm{~m}$ of the ocean (Wells, 1986). In consequence, lower persistence and higher loss of memory of WeMOi and monthly NAO index are to be expected in comparison with monthly AMO index. Also, although WeMOi and monthly NAO index are based on barometric measures at synoptic scales, the effects of the different distances between measurement locations are detected. Whereas WeMOi is defined for a shorter distance (about $1800 \mathrm{~km}$ ), with reference points at San Fernando (Spain) and Padova 541 (Italy), both places in the Western Mediterranean, monthly NAO index is defined covering a 542 larger distance across the Atlantic Ocean (about $3300 \mathrm{~km}$ ), from south-western Iceland to 543 Gibraltar (southern Iberian Peninsula). Thus, different degrees of persistence and loss of 
same for both indices, the effects of the interaction between atmosphere and sea/ocean would be different (Wang et al., 2004; Mosedale, 2004).

547

548 It is also worth mentioning the complexity of the physical mechanism, suggested by the large 549 number of nonlinear equations required for describing every one of the three indices. 550 Whereas $\mu^{*}$ are quite similar for WeMOi and monthly NAO index, without distinction 551 between the different spatial scales, $\mu^{*}$ becomes notably lower for monthly AMO index 552 (based on sea-surface temperatures). In terms of the parameter $\beta$, monthly AMO index 553 could be assumed as a time series with notable persistence (in agreement with its Hurst 554 exponent, $H$ ) and signs of non stationary character. Conversely, WeMOi and monthly NAO 555 index would be qualified as close to stationary uncorrelated series (Malamud and Turcotte, 556 1999). Once again, differences appear between an index based on sea-surface temperatures 557 and the other two based on barometric measures. In spite of these, some common features 558 have to be mentioned: first, a common fractal anisotropy, which is manifested by very 559 similar small values of the Haussdorf exponent, $H_{a}$; second, very similar Lyapunov 560 exponents, $\lambda_{1}$, and Kaplan-Yorke dimensions, $D_{K Y}$. In short, the degree of fractal anisotropy 561 and predictive instabilities for the three indices would be a common feature, becoming 562 physical variables and spatial scales not discriminating factors.

563

564 From the point of view of multifractality, monthly NAO index is close to monofractal 565 behaviour (very narrow spectral width, $W$ ) in comparison with WeMOi and, especially, 566 monthly AMO index. Additionally, the critical Hölder exponent, $\alpha_{0}$, for the monthly AMO 567 index is the highest when comparing it with WeMOi and monthly NAO index. Then, in 568 agreement with definitions of Section 4.2 and contents of Table 1 concerning $\alpha_{0}, W$ and $B$, 569 the fine structure of the physical mechanism resulting from the ocean dynamics governing 570 monthly AMO index would be obtained with more detail than those related to atmospheric 571 dynamics and to interactions between ocean and atmosphere, corresponding to WeMOi and 572 monthly NAO index.

573

574 The simulation of monthly indices by means of the appropriate random series would 575 constitute a possible predictive process. Although the values of the parameters $H, H_{a}$ and $576 \beta$ suggest a $\mathrm{fGn}$ series, MAD and differences between empiric and simulated signals (Table 2 577 and Figure 8) put the stress on the fact that $\mathrm{fGn}$ series are able to simulate some statistical 578 characteristics of the three monthly series, but not a step-by-step prediction. Alternatively, 579 after reviewing the WeMOi fractal and multifractal results and bearing in mind the relative 580 success of the $\mathrm{fGn}$ simulation, an autoregressive process of order $p, A R(p)$, is applied for the 581 prediction of WeMOi. This $\operatorname{AR}(p)$ has to be interpreted as an alternative to a complex system

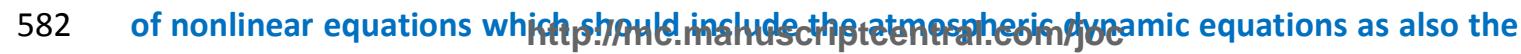


interactions with ocean air masses. This multilinear process would be justified by the

584 moderate persistence, quantified by the Hurst exponent, $H$, and a parameter $\beta$ close to

585 zero. The results of the reconstruction theorem (loss of memory, predictive instability and a

586 not negligible random component of WeMOi), derived without any hypothesis about a

587 specific physical mechanism, are coherent with a high order of the autoregressive process. In

588 short, the adopted approach has consisted on substituting a complex nonlinear system of

589 differential equations by a multilinear regression process, characterised by the required high

590 number of previous empiric WeMOi to predict forthcoming values. In spite of this

591 shortcoming, the ARIMA $(p, 1,0)$ is more efficient than $\mathrm{fGn}$ simulations predicting WeMOi, as

592 proves MAD values obtained for both methods. Whereas $\mathrm{fGn}$ is characterised by a value of

593 MAD close to 3.0 (Table 2), ARIMA(p,1,0) leads to a MAD value close to 1.2 (Figure 10a).

594 Additionally, the ratio between MAD obtained from $\mathrm{fG} n$ and standard deviation of empiric

595 WeMOi (Taula 2) is close to 2.5. Conversely, the ratio between MAD obtained from ARIMA

596 and standard deviation of empiric WeMOi is very close to 1.0. Finally, in agreement with

597 cross-correlations and cross-power spectra derived from WeMO-NAO and WeMO-AMO pairs,

598 monthly NAO and AMO indices would not contribute to an improvement of WeMOi

599 predictions. Only the annual periodicity, a periodicity close to 51 years for the WeMO-AMO

600 pair and another close to half a year for the WeMO-NAO pair are detected.

601

602 

Azorin-Molina C., Lopez-Bustins J.L. (2008). An automated sea breeze selection technique based on regional sea-level pressure difference: WeMOi. Int. J. Climatol., 28, 1681-1692.

Barsugli J.J., Battisti D.S. (1998). The Basic Effects of Atmosphere-Ocean Thermal Coupling on Midlatitude Variability. J. Atmospheric Sciences, 55, 477-492.

Benjamin J.R., Cornell C.A. (1970). Probability, statistics and decision for civil engineering. McGraw-Hill, 685 pp.

Beranová R., Kyselý J. (2015): Links between circulation indices and precipitation in the Mediterranean in an ensemble of regional climate models. Theor. Appl. Climatol., DOI 10.1007/s00704-015-1381-6

Bhatt U.S., Alexander M. (1998). Atmosphere-Ocean Interaction in the North Atlantic: NearSurface Climate Variability. J. Climate, 11, 1615-1632.

Box G.E.P., Jenkins G.M. (1976). Time Series Analysis: Forecasting and Control. Holden-Day, California, 575 pp.

Burgueño A., Lana X., Serra C., Martínez M.D. (2014). Daily extreme temperature multifractals in Catalonia (NE Spain). Physics Letters A, 378, 874-885.

Carlson T.N. (1994): Mid-Latitude Weather Systems. Routledge. London. 507 pp.

Ciasto L.M., Thompson D.W.J. (2004). North Atlantic Atmosphere-Ocean Interaction on Intraseasonal Time Scales. J. Climate, 17, 1617-1621.

Collette C., Ausloos M. (2004). Scaling analysis and evolution equation of the North Atlantic oscillation index fluctuations, Int. J. Mod. Phys. C, 15(10), 1353-1366.

Curry J.A., Webster P.J. (1999): Thermodynamics of atmospheres and oceans. Academic Press. International Geophysics Series, vol. 65, 467 pp.

Czaja A., Frankignoul, C. (1999). Influence of the North Atlantic SST on the atmospheric circulation. Geophys. Res. Letters, 26, 2969-2972.

Dijkstra H.A., Raa L., Schmeits M., Gerrits, J. (2006). On the physics of the Atlantic Multidecadal Oscillation. Ocean Dynamics, 56: 36-50.

Diks C. (1999). Nonlinear time series analysis. Methods and applications. In: Nonlinear time series and chaos, vol 4. World Scientific, London, 209 pp.

Dima M., Lohmann G. (2007). A hemispheric mechanism for the Atlantic Multidecadal Oscillation. J. Climate, 20, 2706-2719.

Dutta S. (2010). EEG pattern of normal and epileptic rats: monofractal or multifractal? Fractals, $18,425-431$.

Eckmann J. P., Oliffson S., Ruelle D., Cilliberto S. (1986). Lyapunov exponents from time series. Phys. Rev. A, 34(6), 4971-4979.

El Kenawy A., López-Moreno J.I., Vicente-Serrano S.M. (2013). Summer temperature extremes in northeastern Spain: spatial regionalization and links to atmospheric circulation (1960-2006). Theor. Appl. Climatol., 113, 387-405. 
659

660

661

662

663

664

665

666

667

668

669

670

671

672

673

674

675

676

677

678

679

680

681

682

683

684

685

686

687

688

689

690

691

692

693

694

695

696

697

698

699

700

701

702

703

704

705

Enfield D.B., Mestas-Núñez A.M., Trimble P.J. (2001): The Atlantic multidecadal oscillation and its relation to rainfall and river flows in the continental U.S. . Geophys. Res. Lett., 28, 20772080.

Feder J. (1988). Fractals. Plenum Press, New York - London.

Feng H., Xu Y. (2012): Multifractal detrended fluctuaction analysis of WLAN. Wireless Pers Commun, 66, 385-395.

Feng T., Zuntao Fu Z., Deng X., Mao J. (2009): A brief description to different multi-fractal behaviors of daily wind speed records over China. Physics Letters A 373, 4134-4141.

Fernández I., Hernández C.N., Pacheco J.M. (2003). Is the North Atlantic Oscillation just a pink noise?, Physica A, 323, 705-714

Ge E., Leung Y. (2012): Detection of crossover time scales in multifractal detrended fluctuation analysis. J Geogr Syst, DOI 10.1007/s10109-012-0169-9.

Ghosh D., Dep A., Dutta S., Sengupta R., Samanta S. (2012). Multifractality of radon concentration fluctuation in earthquake related signal. Fractals, 20, 33-39.

Gonzalez-Hidalgo J.C., Lopez-Bustins J.A., Štepánek P., Martin-Vide J., de Luis M. (2009). Monthly precipitation trends on the Mediterranean fringe of the Iberian Peninsula during the second-half of the twentieth century (1951-2000). Int. J. Climatol., 29, 1415-1429.

Goodman J., Marshall, J. (1999). A Model of Decadal Middle-Latitude Atmosphere-Ocean Coupled Modes. J. Climate, 12, 621-641.

Grassberger P., Procaccia I. (1983a). Characterization of strange attractors, Phys. Rev. Lett., 50, 346-349.

Grassberger P., Procaccia I. (1983b). Estimation of the Kolmogorov entropy from a chaotic signal, Phys. Rev. A, 28, 448-451.

Holton J.R. (2004). An Introduction to Dynamic Meteorology. Elsevier Academic Press, 535 pp.

Hurrell J.W., Kuschnir Y., Visbeck M. (2001). The North Atlantic Oscillation. Science, 291, 603605.

Jones P. D., Jónsson T., Wheeler D. (1997). Extension to the North Atlantic Oscillation using early instrumental pressure observations from Gibraltar and South-West Iceland, Int. J. Climatol., 17, 1433-1450.

Kantelhardt J.W., Zschiegner S.A., Koscielny-Bunde E., Havlin S., Bunde A., Stanley H.E. (2002): Multifractal detrended fluctuaction analysis of nonstationary time series. Physica $A, 316,87-$ 114.

Kaplan J. K., Yorke J. A. (1979). Chaotic behaviour of multidimensional difference equations, in: Functional Difference Equations and Approximation of Fixed Points, edited by: Walter, H. O. and Peitgen, H. O., Springer Verlag, Berlin, 730, 204-227.

Knight J.R., Folland C.H., Scaife A.A. (2006): Climate impacts of the Atlantic Multidecadal Oscillation. Geophysical Research Letters, 33, L17706. 
Koscielny-Bunde E., Kantelhardt J.W, Braund P., Bunde A., Havlin S. (2006): Long-term persistence and multifractality of river runoff records: Detrended fluctuation studies. Journal of Hydrology, 322,120-137.

709

Latif M. (1998). Dynamics of Interdecadal Variability in Coupled Ocean-Atmosphere Models. J. Climate, 11, 602-624.

Lionello P., Malanotte-Rizzoli P., Boscolo R., (Eds) (2006): Mediterranean climate variability. Developments in Earth and Environmental Sciences, 4. Elsevier, Amsterdam, 421 pp.

López-Bustins J.A., Martín-Vide J., Sánchez-Lorenzo A. (2008): Iberia winter rainfall trends based upon changes in teleconnection and circulation patterns. Global and Planetary Change, $63,171-176$.

719

López-Moreno J.I., Vicente-Serrano S.M., Morán-Tejeda E., Lorenzo-Lacruz J., Kenawy A.,

Mak M. (2011). Atmospheric Dynamics. Cambridge University Press, New York, 486 pp.

Malamud B.D., Turcotte D.L. (1999). Self-affine time series: Generation and analyses. Advances

Mali P. (2014). Multifractal characterization of global temperature anomalies. Theor. Appl. Climatol., DOI 10.1007/s00704-014-1268-y.

Mariotti A., Dell'Aquila A. (2012). Decadal climate variability in the Mediterranean region: roles of large-scale forcings and regional processes. Clim. Dyn., 38, 1129-1145.

Martin J.E. (2006). Mid-latitude Atmospheric Dynamics. A first course. John Wiley \& Sons, $324 \mathrm{pp}$.

Martín M.L., Luna M.Y., Morata A., Valero F. (2004). North Atlantic teleconnection patterns of low-frequency variability and their links with springtime precipitation in the western Mediterranean. Int. J. Climatol., 24, 213-230.

Martín-Vide J., López-Bustins J.A. (2006). The Western Mediterranean Oscillation and rainfall in the Iberian Peninsula. Int. J. Climatol., 26, 1455-1475.

Martín-Vide J., Sánchez-Lorenzo A., López-Bustins J.A., Cordobilla M.J., Garcia-Manuel A., Raso J.M. (2008). Torrential rainfall in northeast of the Iberian Peninsula: synoptic patterns and WeMO influence. Adv. Sci. Res., 2, 99-105.

Martínez M.D., Lana X., Burgueño A, Serra C. (2010). Predictability of monthly North Atlantic Oscillation index based on fractal analyses and dynamic systems theory. Nonlinear Process Geophys., 17, 93-101.

Mosedale T. J. (2004). North Atlantic Ocean-Atmosphere Interaction using Simple and Complex Models. PhD Thesis, Department of Meteorology. Reading University, UK, 195 pp.

Mosedale T.J., Stephenson D.B., Collins M. (2005). Atlantic Atmosphere-Ocean Interaction: A Stochastic Climate Model-Based Diagnosis. J. Climate, 18, 1086-1095.

Mosedale T.J., Stephenson D.B., Collins M., Mills T.C. (2006). Granger Causality of Coupled Climate Processes: Ocean Feedback on the North Atlantic Oscillation. J. Climate, 19, 11821194. 
761

762

763

764

765

766

767

768

769

770

771

772

773

774

775

776

777

778

779

780

781

782

783

784

785

786

787

788

789

790

791

792

793

794

795

796

797

798

799

800

801

802

803

Movahed M. S., Hermanis E. (2008): Fractal analysis of river flow fluctuations. Physica A, 387, 915-932.

Muzy J.F., Bacry E., Arneodo A. (1994): The multifractal formalism revisited with wavelets. Internat. J. Bifur. Chaos, 4, 245-302.

Ouachani R., Bargaoui Z., Ouarda T. (2013). Power of teleconnection patterns on precipitation and extreme flow variability of upper Medjerda Basin. Int. J. Climatol., 33, 5876.

Press W.H., Teukolsky S.A., Vetterling W.T., Flannery B.P. (1992). Numerical recipes in FORTRAN, $2^{\text {ed }}$. Cambridgwe University Press, Cambridge.

Ríos-Cornejo D., Penas A., Álvarez-Esteban R., Del Río S. (2015). Links between teleconnection patterns and mean temperature in Spain. Theor. Appl. Climatol., DOI 10.1007/s00704-014-1256-2.

Sánchez-Lorenzo A., Calbó J., Brunetti M., Deser C. (2009) : Dimming/brightening over the Iberian Peninsula: trends in sunshine duration and cloud cover and their relation with atmospheric circulation. J. Geoph. Res., 114, D00D09.

Shimizu Y., Thurner S., Ehrenberger K. (2002): Multifractal spectra as a measure of complexity in human posture. Fractals, 10, 103-116.

Stephenson D.B., Pavan V., Bojariu R. (2000). Is the North Atlantic Oscillation a random walk? Int. J. Climatol., 20, 1-18.

Stoop F., Meier P.F. (1988). Evaluation of Lyapunov exponents and scaling functions from time series, J. Opt. Soc. Am. B, 5, 1037-1045.

Trigo R.M., Osborn T.J., Corte-Real J.M. (2002). The North Atlantic Oscillation influence on Europe: climate impacts and associated physical mechanisms, Clim. Res., 20, 9-17.

Turcotte D.L. (1997). Fractals and Chaos in Geology and Geophysics (2 ${ }^{\text {nd }}$ Edition). Cambridge University Press, Cambridge, UK, 398 pp.

Vicente-Serrano S.M., Beguería S., López-Moreno J.I., El Kenawy A.M., Angulo-Martínez M. (2009). Daily atmospheric circulation events and extreme precipitation risk in northeast Spain: Role of the North Atlantic Oscillation, the Western Mediterranean Oscillation, and the Mediterranean Oscillation. J. Geophys. Res., 114(D8), DOI: 10.1029/2008JD011492.

Wallace J.M., Smith C., Jiang Q. (1990). Spatial patterns of Atmosphere - Ocean interaction in the Northern winter. J. Climate, 3, 990-998.

Wang C., Xie S.P., Carton J.A. (2004). A global survey of ocean-atmosphere and climatic variability. In Earth climate: The Ocean-Atmosphere interaction. Geophys. Monograph, 147, American Geophysical Union, Washington D.C., 1-19.

Wells N. (1986). The atmosphere and ocean: A physical introduction. Taylor \& Francis, London, $347 \mathrm{pp}$.

Wiggins S. (2003). Introduction to applied nonlinear dynamical systems and chaos ( $2^{\text {nd }}$ edition). Text in Applied Mathematics, 2. Springer, New York, 843 pp. 
804 Xin X., Xue W., Zhang M., Li H., Zhang T., Zhang J. (2015). How much of the NAO monthly 805 variability is from ocean - atmospheric coupling: results from an interactive ensemble 806 climate model. Climate Dynamics, 44, 781-790. 


\begin{tabular}{|c|c|c|c|c|c|c|c|c|c|c|}
\cline { 2 - 11 } \multicolumn{1}{c|}{} & \multicolumn{9}{c|}{ Monofractal parameters } & \multicolumn{3}{c|}{ MF-DFA } \\
\cline { 2 - 11 } \multicolumn{1}{c|}{} & $H$ & $H_{a}$ & $\beta$ & $\mu^{*}$ & $\kappa$ & $\lambda_{1}$ & $D_{K Y}$ & $\alpha_{o}$ & $B$ & $W$ \\
\hline WeMO & $0.67(0.49)$ & 0.05 & 0.17 & 9.68 & 0.64 & 0.16 & 12.43 & 0.57 & 1.01 & 0.23 \\
\hline NAO & $0.53(0.56)$ & 0.10 & 0.07 & 10.1 & 1.37 & 0.13 & 12.53 & 0.56 & -0.01 & 0.07 \\
\hline AMO & $0.91(0.99)$ & 0.10 & 1.06 & 6.62 & 0.24 & 0.15 & 12.15 & 1.03 & 1.26 & 0.50 \\
\hline
\end{tabular}

2

3 Table 1. Mono- and multifractal parameters of the monthly WeMO, NAO and AMO indices. 4 Hurst exponents within parentheses are those derived from multifractal analyses, under the 5 assumption that monthly series are stationary.

6 
Page 29 of 42

8

$\begin{array}{ll}1 & 9 \\ 2 & \\ 3 & \\ 4 & \\ 5 & \\ 6 & \\ 7 & \\ 8 & \\ 9 & \\ 10 & \\ 11 & \\ 12 & 10 \\ 13 & 11 \\ 14 & 12 \\ 15 & 13 \\ 16 & 14 \\ 17 & 15 \\ 18 & 16 \\ 19 & 17 \\ 20 & \end{array}$

International Journal of Climatology - For peer review only

\begin{tabular}{|c|c|c|c|c|c|c|}
\cline { 2 - 7 } \multicolumn{1}{c|}{} & Range & $<$ diff $>$ & $\sigma($ diff) & K-S test & MAD & $\sigma(e m p)$ \\
\hline WeMO & $(-4.36,6.41)$ & 0.42 & 1.55 & $\begin{array}{c}0.03 \\
(0.05)\end{array}$ & 2.94 & 1.17 \\
\hline NAD & $(-6.99,6.62)$ & 0.15 & 2.44 & $\begin{array}{c}0.03 \\
(0.05)\end{array}$ & 1.85 & 1.72 \\
\hline HMO & $(-0.76,0.88)$ & 0.01 & 0.24 & $\begin{array}{c}0.02 \\
(0.05)\end{array}$ & 0.28 & 0.21 \\
\hline
\end{tabular}

Table 2. Parameters describing the differences between empiric monthly indices and those simulated by $\mathrm{fG}$ series. Columns correspond to range, average, $\langle$ def $\rangle$, and standard deviation, $\sigma($ diff), Kolmogorov-Smirnov statistics for a Gaussian distribution ( $95 \%$ confidence level within parentheses), mean absolute deviation, MAD, and the standard deviations, $\sigma(\mathrm{emp})$, of the empiric monthly signals.

2

http://mc.manuscriptcentral.com/joc 
(a)

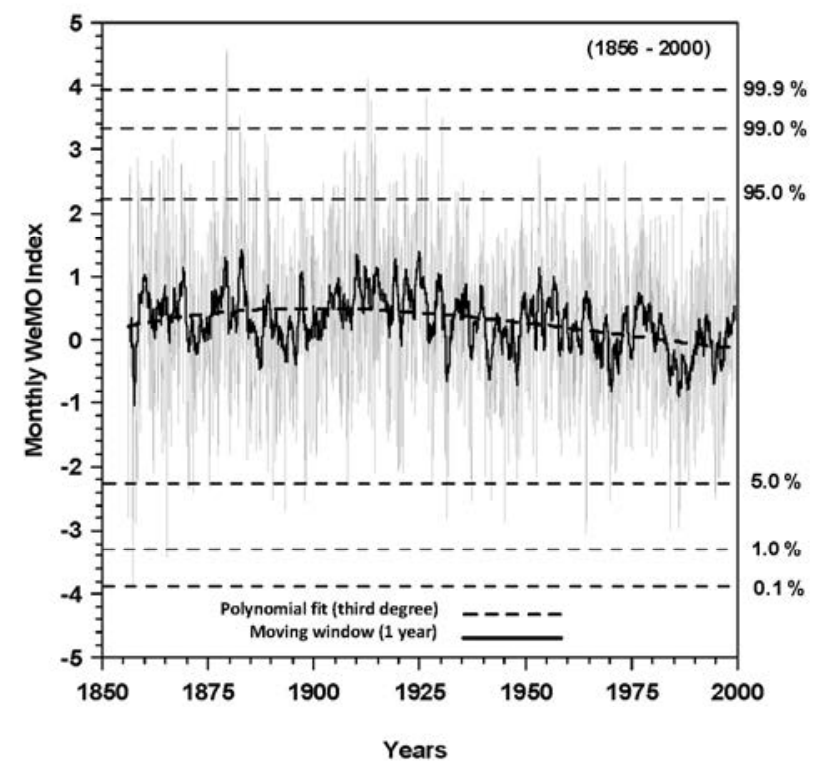

$210 \times 297 \mathrm{~mm}(200 \times 200 \mathrm{DPI})$

http://mc.manuscriptcentral.com/joc 
14 (b)
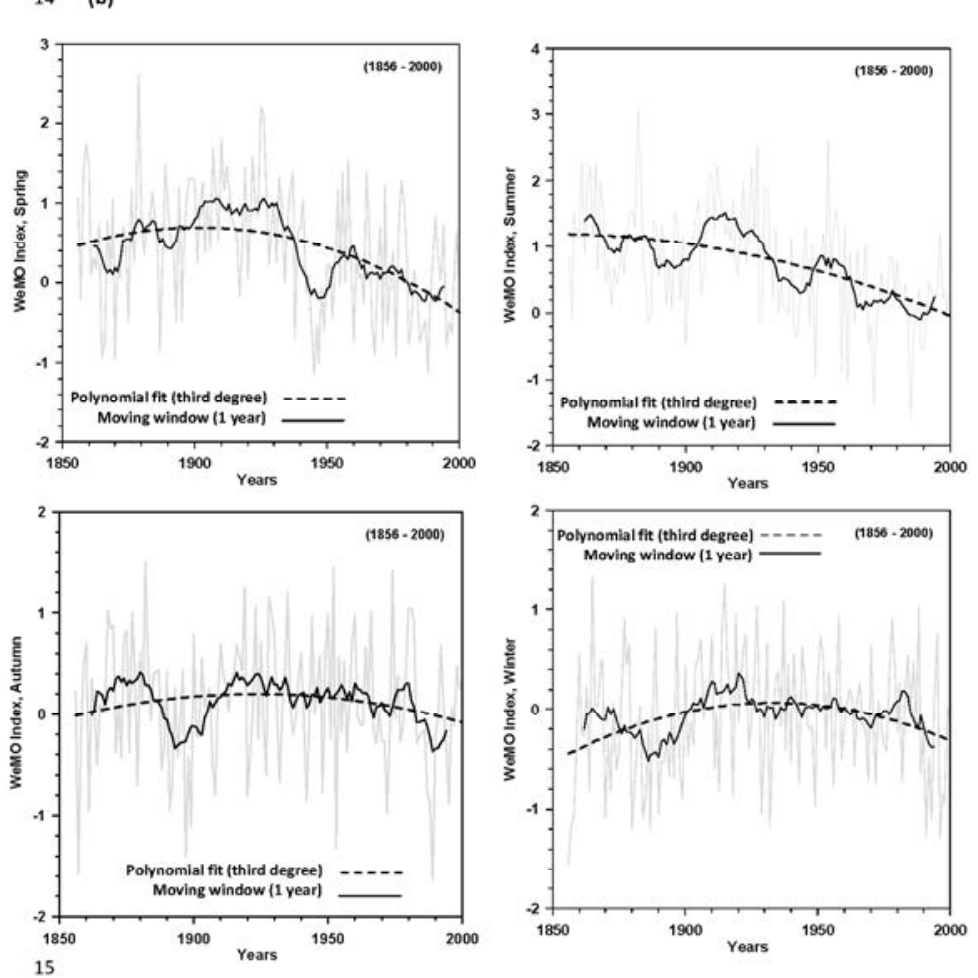

16

17

18

19

21 Figure 1. Evolution of the WeMOi along years $1856-2000$ at (a) monthly scale, smoothed by a moving window of 13 -months length. The cumulative distribution of WeMOi is labelled at right. (b) WeMOi grouped by seasons (JFM stands for January, February and March, and so on with AMJ, JAS and OND). 
55

56

57

58

59

60

61

62

63

64

65

66

67

68

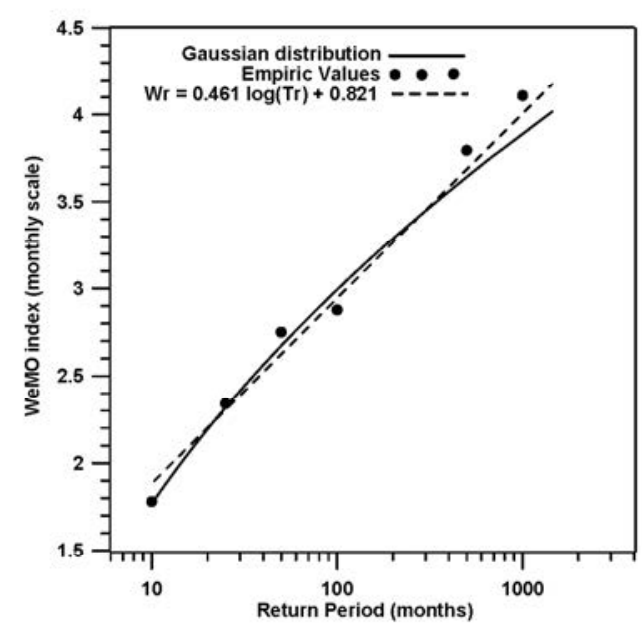

Figure 3. Expected values of WeMOi derived for different return periods, compared with empiric values.

$210 \times 297 \mathrm{~mm}(200 \times 200 \mathrm{DPI})$

http://mc.manuscriptcentral.com/joc 
$72 \quad$ a)

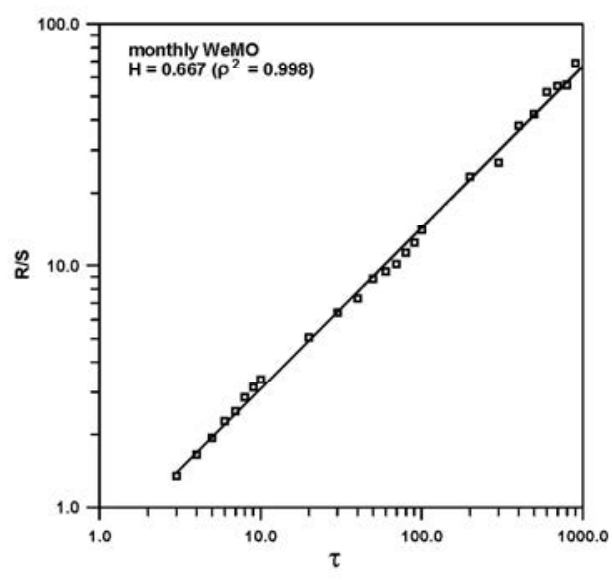

73

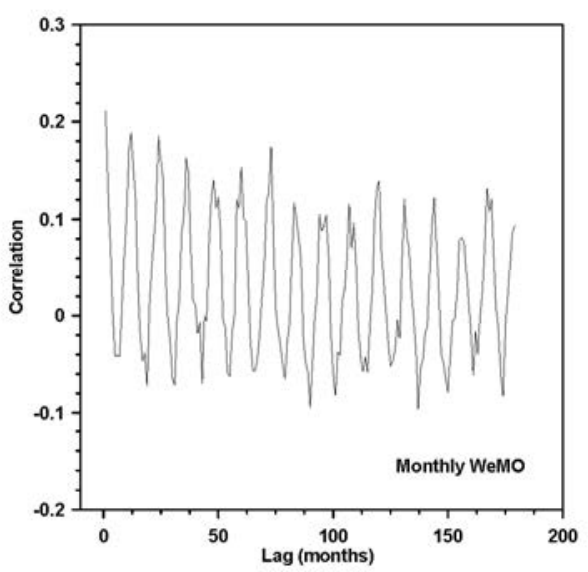

4 b)

75

76

77

$210 \times 297 \mathrm{~mm}(200 \times 200$ DPI $)$

http://mc.manuscriptcentral.com/joc 
$78 \quad$ c)

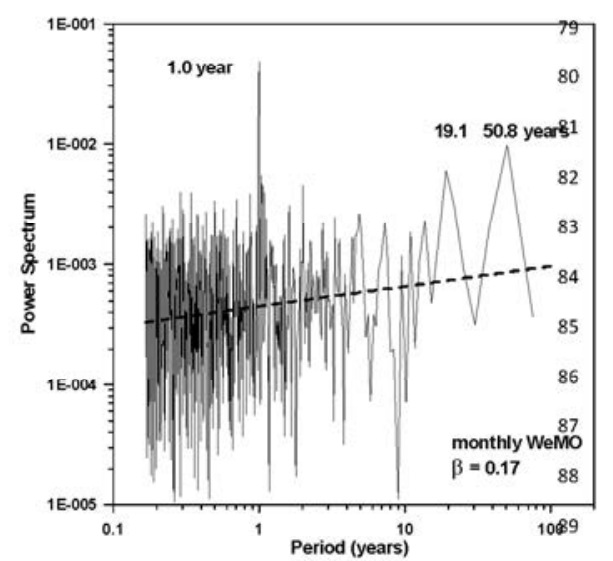

90

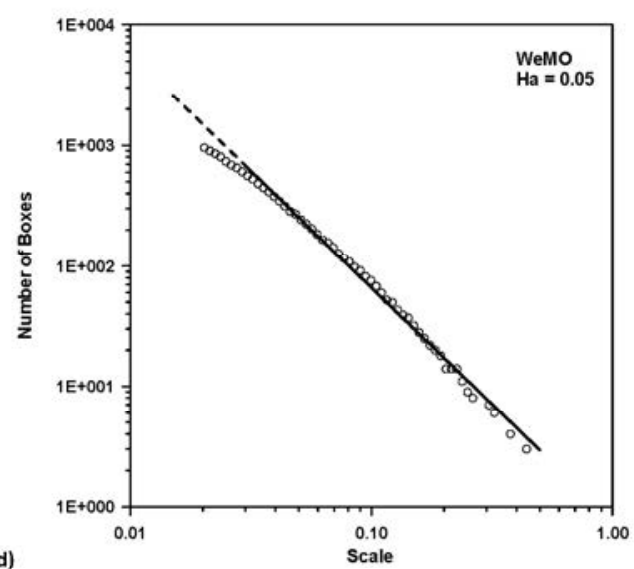

91 d)

92

93 Figure 4. Monofractal analysis of the WeMOi a) Hurst exponent; b) autocorrelation; c) power

94 spectrum and d) Hausdorff exponent deduced from the box-counting algorithm.

$$
6
$$

$210 \times 297 \mathrm{~mm}(200 \times 200 \mathrm{DPI})$ 


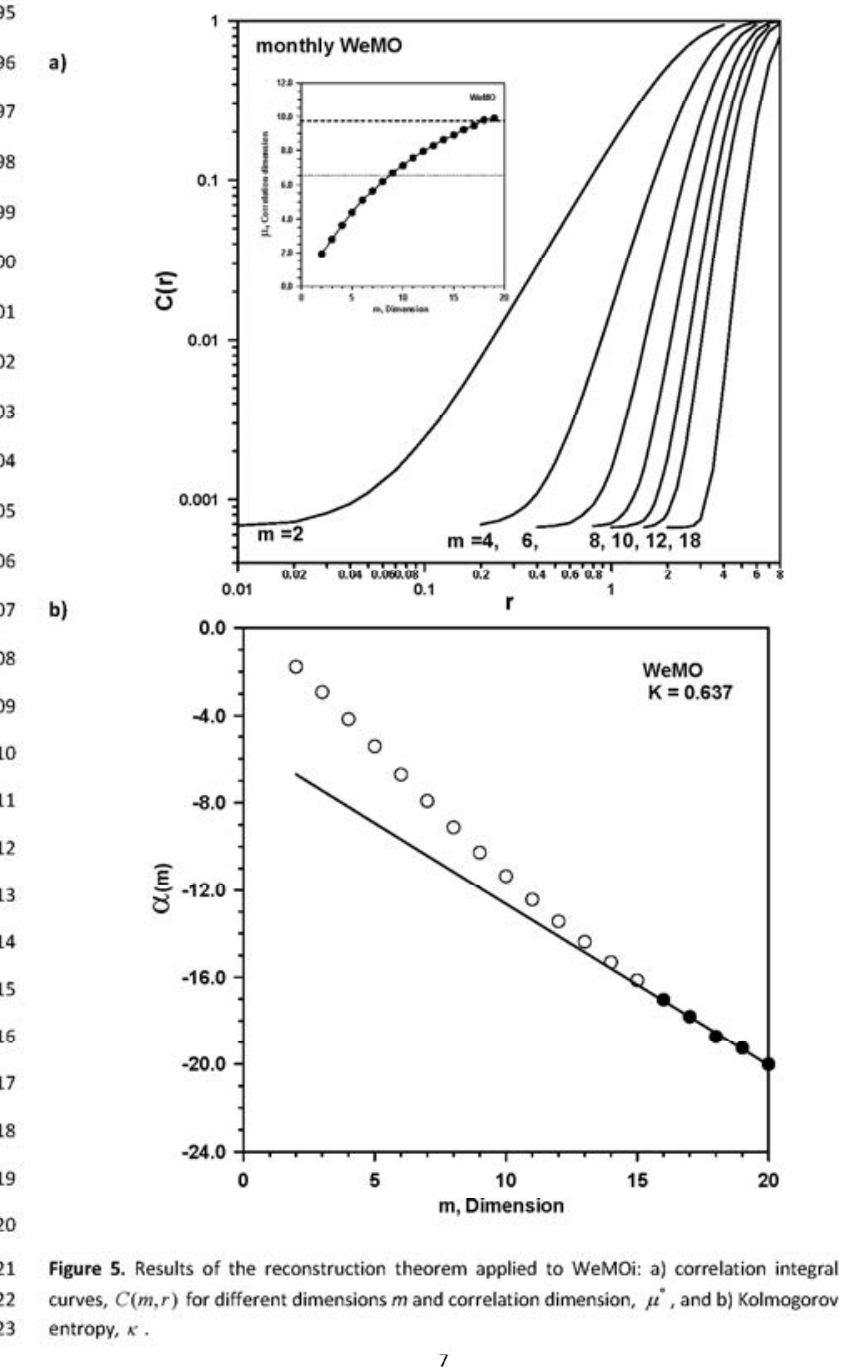

$210 \times 297 \mathrm{~mm}(200 \times 200 \mathrm{DPI})$ 
a)

b)
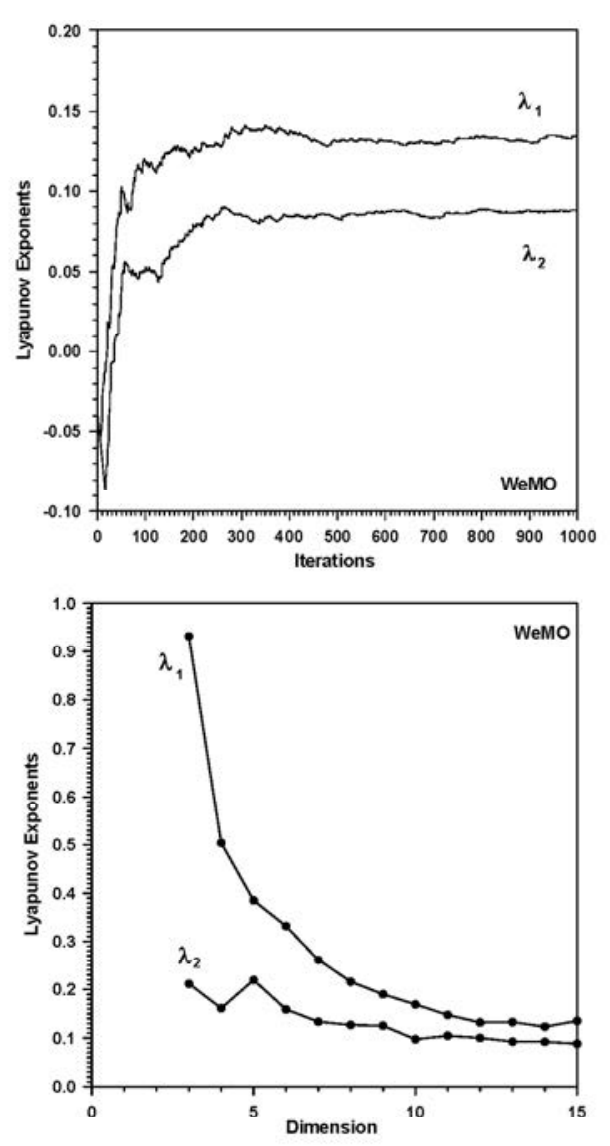

Figure 6. Evolution of the first two Lyapunov exponents with a) the increasing number of iterations and $b$ ) the reconstruction dimension $m$.

$210 \times 297 \mathrm{~mm}(200 \times 200$ DPI $)$

http://mc.manuscriptcentral.com/joc 
148

a)

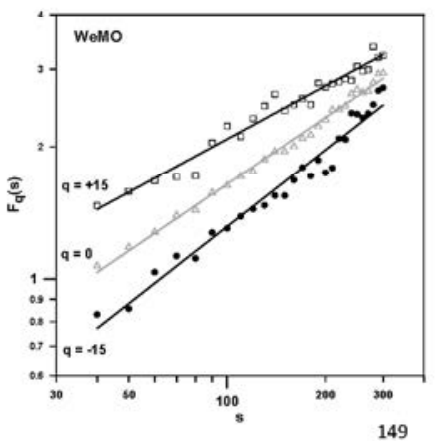

150

151

152

153

154

155

156

157 b)

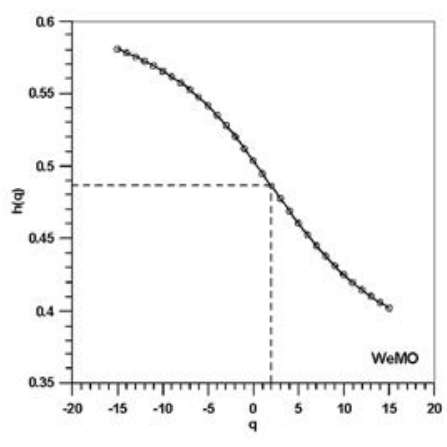

d)

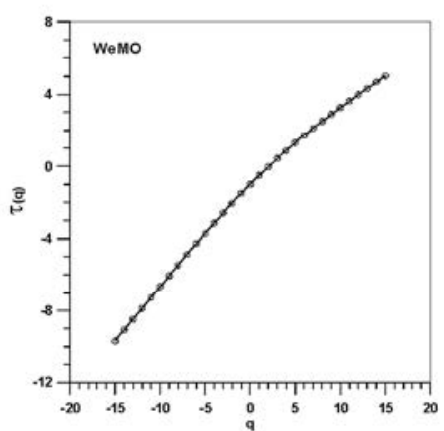

10

$210 \times 297 m m(200 \times 200$ DPI $)$

http://mc.manuscriptcentral.com/joc 
a)

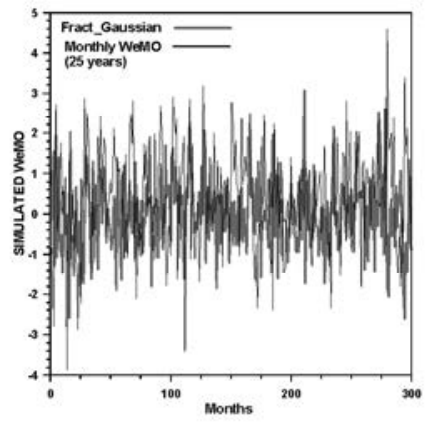

b)

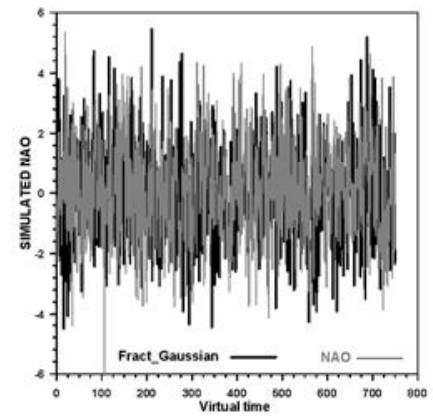

c)

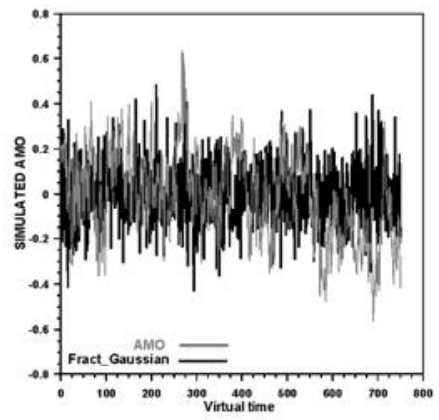

Figure 8. Comparison, through 25 years, between simulated $\mathrm{fG}$ and empiric monthly series of a) WeMO, b) NAO and c) AMO indices.

12

$210 \times 297 \mathrm{~mm}(200 \times 200$ DPI $)$

http://mc.manuscriptcentral.com/joc 

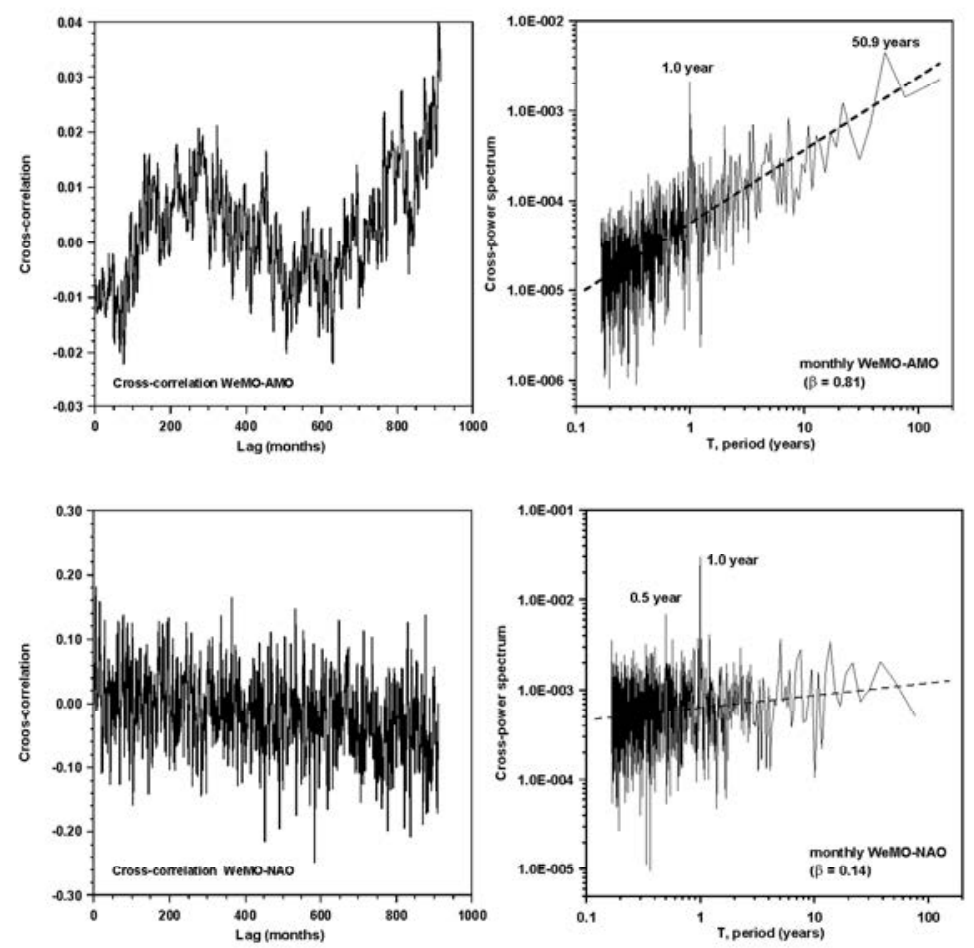

Figure 9 Figure 9. Cross-correlations and cross-power spectra for pairs WeMO-AMO and WeMO-NAO indices.

13

$210 \times 297 \mathrm{~mm}(200 \times 200 \mathrm{DPI})$ 
a)

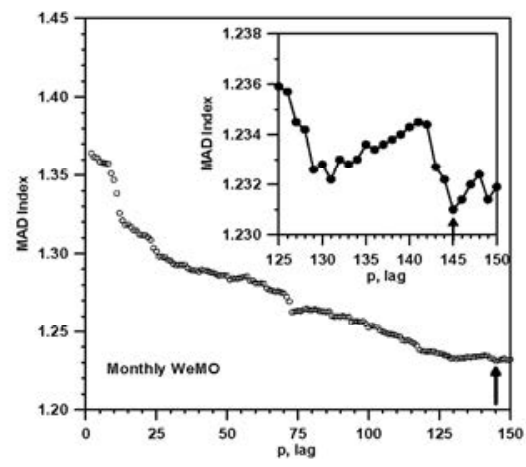

b)

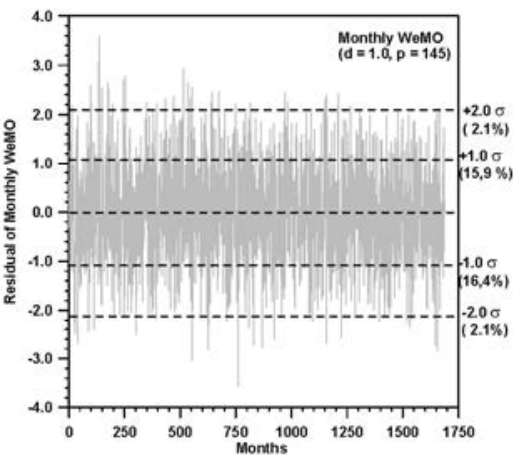

c)

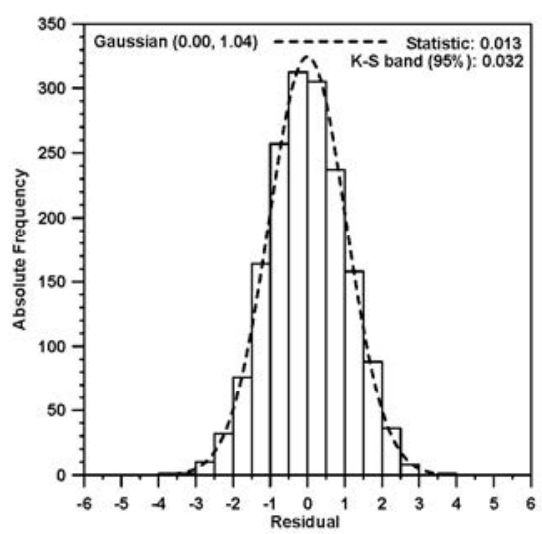

Figure 10. a) Residuals of the autoregressive process for the whole recording period of the WeMOi. b) Evolution of MAD with the autoregression order, $p$. c) Histogram of WeMOi residuals.

$$
14
$$

$210 \times 297 \mathrm{~mm}(200 \times 200 \mathrm{DPI})$

http://mc.manuscriptcentral.com/joc 